\title{
Increasingly microphysiological models
}

\author{
Modelling human tissues in microphysiologically relevant 'chips' will increasingly help to unravel mechanistic \\ knowledge underlying disease, and might eventually accelerate the productivity of drug development and predict \\ how individual patients will respond to specific drugs.
}

A

bout $90 \%$ of candidate drugs fail human trials, largely because of lack of evidence of efficacy or, to a lesser extent, because of unacceptable toxicity. Notwithstanding continuous advances in technology for in vitro culture and in computational modelling and data analysis, and despite the widening availability of better animal models, the rate of drug approvals per inflation-adjusted researchand-development spending in the United States has been progressively dwindling for many decades.

Clearly, from a translational lens, preclinical testing is largely failing. Cell lines, cells in tissue-culture plates and cells kept in non-physiological environments do not behave as they do in the body. Rodents can be useful disease models, but they mostly fail to recapitulate the characteristics of disease in humans. Drug testing in nonhuman primates and disease models in large animals are limited and expensive, and they can also be unrepresentative of disease physiology and drug pharmacology observed in patients.

Human-relevant in vitro modelling at the tissue and organ levels would clearly be advantageous. Piggybacking on microfabrication techniques (in particular, replica moulding, soft lithography and microcontact printing), tissue engineering and stem cell biology, sophisticated systems that recreate the biology of human tissue in vitro - in particular, the so-called tissue chips or organs-on-chips - are being developed in academia and in industry. The development of tissue chips has been spurred and supported by government initiatives, such as the Tissue Chip Program of the National Center for Advancing Translational Sciences (part of the United States National Institutes of Health) and the 'Organ-on-Chip in Development project' within the European Union's Horizon 2020 research and innovation programme.

As somewhat minimalist yet controllable engineered microsystems, tissue chips recapitulate relevant features of the native dynamic microenvironment of cells. Designing such microphysiological systems, therefore, involves finding the minimal set of features - involving device architecture, tissue components, tissue microstructure,
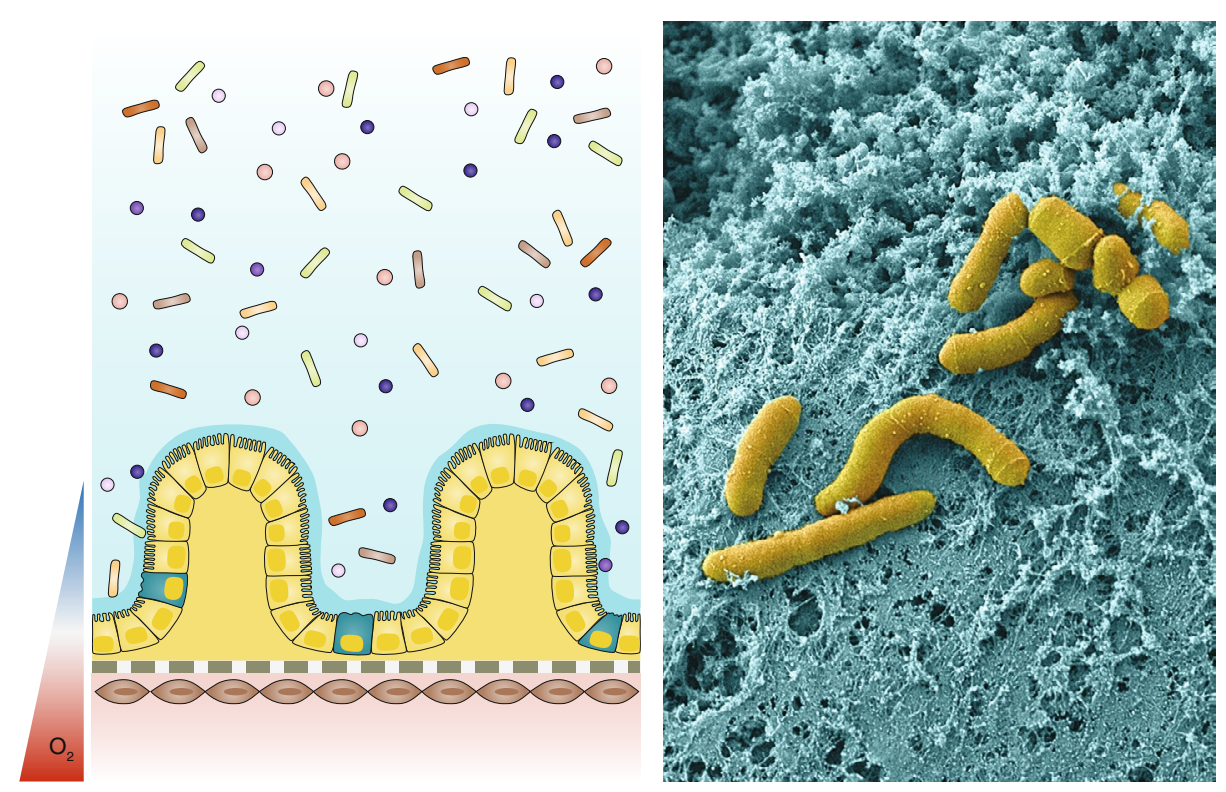

Fig. 1 | Schematic of a microphysiological system mimicking the human-gut microbiome, and scanning electron micrograph of commensal bacteria on a layer of secreted mucous above the apical surface of the epithelium in the microphysiological system. Left panel reproduced from Poceviciute, R. \& Ismagilov, R. F. Nat. Biomed. Eng. https://doi.org/10.1038/s41551-019-0425-0 (2019). Right panel Credit: Wyss Institute at Harvard University

flow characteristics, interactions between cell types and environmental gradients, among others - that are necessary to reproduce particular functions of human organs. Since a two-chamber lung-on-a-chip that mimicked the inhalation and exhalation of the lung alveolus (by allowing for gas exchange between endothelial and alveolar epithelial cells) was reported in 2010 (Huh, D. et al. Science 328, 1662-1668; 2010), many other tissue chips have faithfully modelled the physiology of human tissues. These chips are increasingly modular, allow for a diversity of functional readouts, can be interrogated in situ frequently and at high resolution, and most recently are being vascularly interconnected to form a humanbody-on-a-chip for the study of drug pharmacokinetics and pharmacodynamics across tissues.

As exemplified by four Articles included in this issue, tissue chips are increasingly recapitulating more complex tissue (patho) physiology. By incorporating physiologically relevant oxygen gradients, Donald Ingber and colleagues leveraged their previously developed microfluidic intestine-on-achip to maintain, for a few days, a culture of living human intestinal epithelium with stable communities of aerobic and anaerobic human gut microbiota (Fig. 1). The prolonged culture and the diversity of microorganisms cultured in the chip should enable the study of functional interactions between a patient's intestinal epithelial cells and commensal gut microorganisms.

In addition to fluid flow, tissue chips can incorporate necessary mechanical motion, such as peristalsis-like stretching in the intestine chip. Another example of the importance of properly modelling the mechanics of tissues, reported by Andrea Barbero and colleagues, is the need for hyperphysiological compression in a microphysiological cartilage-on-a-chip in order for it to recapitulate the pathogenesis of osteoarthritis (such as inflammation and hypertrophic traits). The device may serve 
as a tool for the study of the mechanisms that lead to the progressive degeneration of articular cartilage in patients with osteoarthritis. Microphysiological systems can also make it easier to study the interplay of mechanical and biochemical factors in disease, as shown by Andre Levchenko, Steven An, Onur Kilic and colleagues with a microphysiological model of the bronchial airways. The researchers found that bronchial spams (sudden constrictions of the airways that occur in asthma and chronic bronchitis) result from mechanochemical feedback loops between smooth muscle cells and epithelial cells.

All these microphysiological systems could, in principle, be used to screen drugs and to determine how a patient would respond to a particular drug.
Yet the predictive possibilities of these microphysiological systems largely remain to be validated with clinical data. An early attempt is described by Dong-Woo Cho, Sun Ha Paek and colleagues, who designed a simple tumour-on-a-chip model employing printed bioinks containing patient-derived glioblastoma cells, human vascular endothelial cells and decellularized extracellular matrix from porcine brain tissue to identify, in seven patients with glioblastoma, their resistance to the standard treatment (radiotherapy and the administration of the chemotherapeutic temozolomide). The chip qualitatively replicated the treatment responses of the patients.

Thorough benchmarking against clinical data will be necessary before tissue chips can be used for the toxicological assessment of drugs, for drug-efficacy testing and for the prediction of patients' responses to drugs. Ideally, a patient-on-a-chip system would only use cells derived from a single line of patient-derived induced pluripotent stem cells, achieve matured tissues with stable phenotypes, use inert materials, have separated tissue and vascular compartments and functionally scaled tissues, and allow for multiple online readouts (structural, molecular and functional) in longitudinal studies. Innovation and collaborations across academia and industry will eventually get us there.

Published online: 5 July 2019

https://doi.org/10.1038/s41551-019-0433-0 